\title{
Performance of Partially Fluorinated Polyimide Insulation for Aerospace Applications
}

Ahmad N. Hammoud and Mark W. Stavnes

NYMA, Inc.

Brook Park, Ohio

James R. Ide and Ed Muegge

McDonnell Douglas Aerospace

St. Louis, Missouri

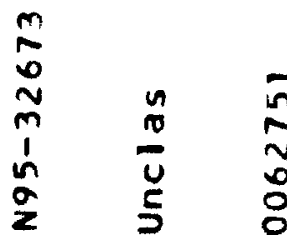

August 1995

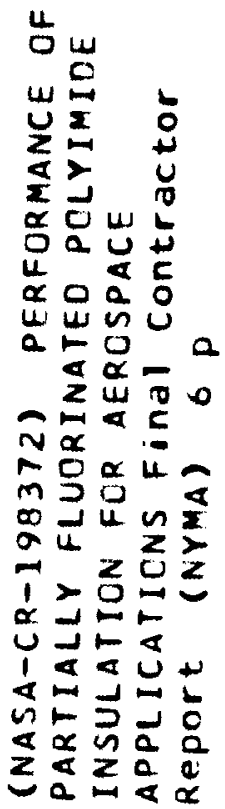

Prepared for

Lewis Research Center

Under Contracts NAS3-27186 and NAS1-18580

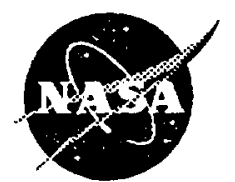

National Aeronautics and Space Administration 



\section{Performance of Partially Fluorinated Polyimide Insulation for Aerospace Applications}

\author{
Ahmad N. Hammoud and Mark W. Stavnes \\ Nyma, Inc. \\ NASA Lewis Research Center \\ 2001 Aerospace Parkway \\ Brook Park, Ohio 44142
}

\author{
James R. Ide and Ed Muegge \\ McDonnell Douglas Aerospece \\ P.O. Box 516 \\ St. Louis, MO 63166
}

\begin{abstract}
Polyimide has been used extensively as the primary wiring insulation in commercial planes, military aircraft and space vehicles due to its low weight, high service temperature and good dielectric strength. New failure modes, however, have been associated with the use of polyimide because of the susceptibility of the insulation to pyrolization and arc tracking. A new wiring construction utilizing partially fluorinated polyimide insulation has been tested and compared with the standard military polyimide wire. Electrical properties which were investigated include AC corona inception and extinction voltages (sea level and 60,000 feet), time/current to smoke and wire fusing time. The two constructions were also characterized in terms of their mechanical properties including abrasion resistance, dynamic cut through and notch propagation. In this paper, these test efforts and the results obtained are presented and discussed.
\end{abstract}

\section{Introduction}

Polyimide based wiring insulation (MII-W-81381) is widely used in electrical wiring systems for aerospace applications due to its high dielectric strength, low weight, non-flammability and high temperature capability. New failure modes, however, have been reported by the aerospace community in which degraded polyimide insulation becomes conductive upon an initiation. These failures are detrimental to mission completion and safety as they have proven to be very costly in terms of loss of life and expensive equipment.

A NASA program whose objective is to increase the safety of spacecraft wiring systems is being managed at NASA LeRC. The program uses a database of testing information on various candidates to replace MIL-W. 81381, and identifies the requirements which are relevant to the NASA missions. Specifically, an extensive testing database was developed by the Air Force Wright Laboratory under contract with McDonnell Douglas Aerospace (MDA) which thoroughly addressed the aircraft operational environments [1]. By determining the operational environments for NASA, as compared to the aircraft environments, the additional operational requirements for NASA missions were determined and unique tests

to be performed were revealed [2].

An experimental insulation material developed by TRW under the Air Force's More Electric Aircraft contract, exhibited some very promising electrical and mechanical properties for aerospace applications. This insulation material uses a partially fluorinated polyimide (PFPI) formulation and exhibits similar mechanical and electrical properties to polyimide material without the arc propagation tendencies. In this work, PFPI insulation was characterized according to SAE AS-4373 standards in terms of the following:

Corona Inception/Extinction

Wire Fusing Time

Dynamic Cut Through

Weight Loss/Outgassing

The NASA test program compared 20 AWG samples of MIL-W-81381/ $/$ and PFPI insulated wire produced by TRW. The TRW sample was delivered with a solid conductor instead of stranded. This reduced the flexibility of the sample and promoted cracking in the PFPI layers. Since the PFPI material is in the developmental stages, the process of applying the material to the conductor has not been perfected. The process used to produce the samples permitted air pockets to form in the insulated layers degrading the materials performance. The conductor experienced tensile stress and flexure during processing which led to the development of micro cracks forming in the insulated layers. During the test, extreme care was taken in the preparation and handling of the PFPI samples in order to produce reliable results.

\section{PFPI/MIL-W-81381 Test Results}

\section{Corona Inception and Extinction Test}

A $400 \mathrm{~Hz}$ power source was used to determine the AC corona inception and extinction voltages. Tests were performed on wire samples that were exposed to ambient temperature at pressures of sea level and 60,000 feet. Three specimens of each wire sample were wrapped for 10 equally spaced turns around a $10 \mathrm{X}$ mandrel. Power was applied at a rate of $\mathbf{5 0}$ volts per second until continuous corona discharges were observed on the positive half cycle of the AC waveform. After the inception voltage was determined, 
the voltage was lowered gradually until the discharges were no longer detectable and this voltage was reconded as the extinction voltage.

Corona results at sea level are shown in Figure 1 and results at 60,000 feet are shown in Figure 2. In the test, the inception and extinction levels for the MIL-W81381 specimens were consistently $140 \mathrm{~V}_{\mathrm{rm}}$ higher than those of the PFPI construction. The differential voltage required to extinguish the corona was comparable for both constructions at $70 \mathrm{~V}_{\mathrm{mms}}$.

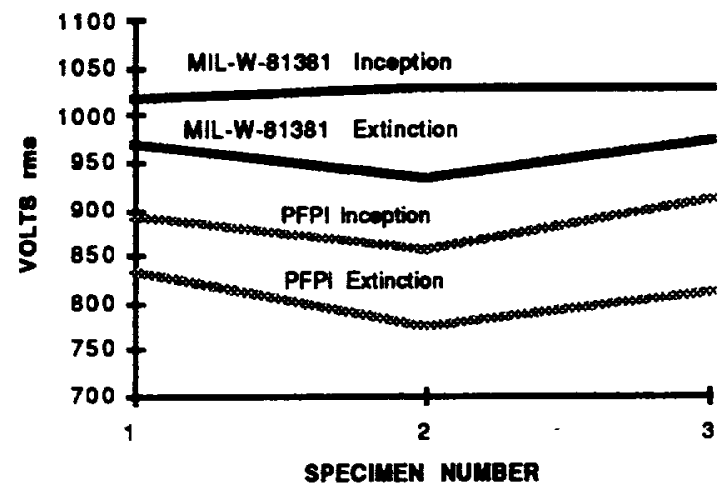

Figure 1. Corona Test Results at Sea Level.

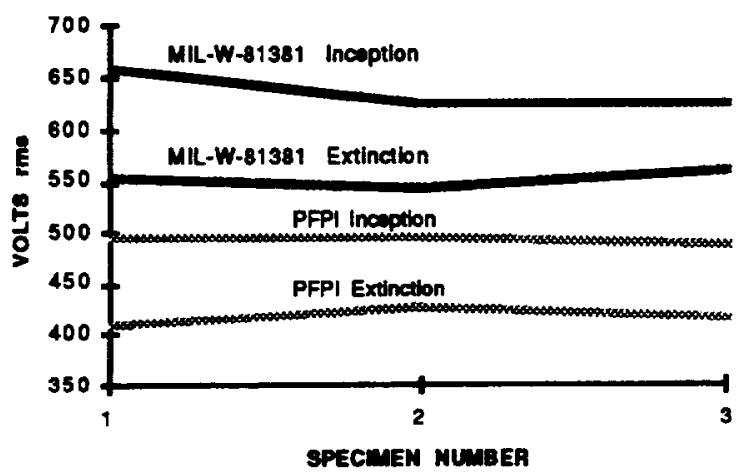

Figure 2. Corona Results at Altitude.

\section{Time/Current to Smoke Test}

The time/current to smoke test was used to determine the current and duration required to produce smoke from a finished wire specimen. Six samples of each wire type were used in this test. The test began by raising the current through the specimen to 10 amps. Once 10 amps of current was achieved, a stop watch was started. After 30 seconds, the current was increased 5 amps for an additional 30 seconds. This process was repeated until smoke was detected.

The results of the time/current to smoke test are shown in Figure 3. All of the MIL-W-81381 samples survived 40 amps. One sample failed at 45 seconds, four at 50 seconds and one at 55 seconds with the average being 10.2 seconds at 50 amps. The PFPI samples did not perform as well. Two samples failed at 35 seconds while the other four samples failed at 40 seconds with the average being 0.2 seconds at 40 amps. It should be noted that the smoke on the MII-W-81381 wire vented out of the seams of the tape construction while the PFPI construction vented from the bubbles and imperfections present in the insulation.

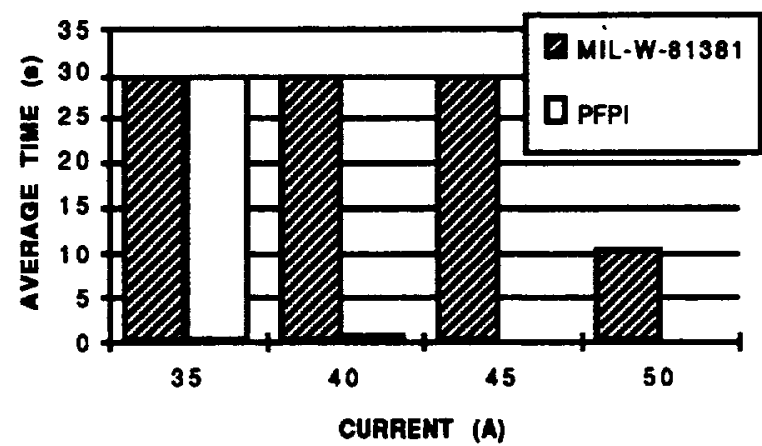

Figure 3. Time/Current to Smoke Test Results.

\section{Wire Fusing Time Test}

The wire fusing time test was used to determine the time required to interrupt an insulated wire sample during an overcurrent condition. Six samples of each wire type were used in this test. A DC constant current supply was set for 60 amps which is 2.5 times the rated current of $20 \mathrm{AWG}$ silver plated copper conductor. The current was applied to the specimen and the time to interrupt was recorded.

The results of the wire fusing time test are shown in Figure 4. The MIL-W-81381 samples averaged 24.8 seconds to interrupt while the PFPI samples averaged 14.4 seconds. It should be noted that all the samples tested were fairly consistent across the specimen base. The MII-W-81381 wire samples averaged 10.4 seconds longer to intemupt in the overcurrent condition than $d d$ the TRW PFPI samples. The PFPI construction started venting out of the bubbles and imperfections present in the insulation within a few seconds of power being applied. The disparity in performance between the two specimens can be partially attributed to the immediate breakdown of the PFPI insulation through the imperfections and partially due to the utilization of solid conductor in the PFPI construction. 


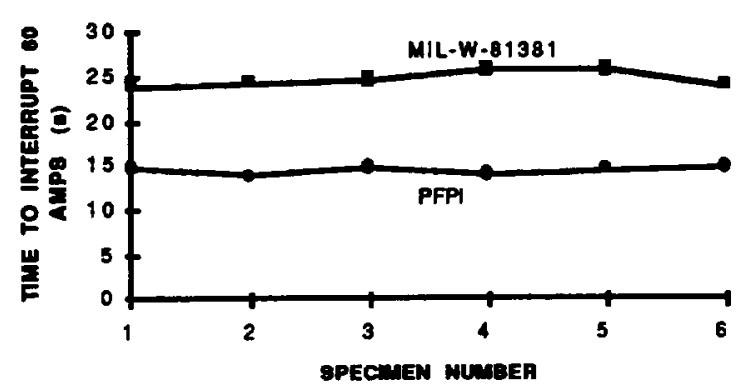

Figure 4. Wire Fusing Time Test Results.

\section{Abrasion Resistance Test}

The abrasion test was used to provide a relative abrasion resistance evaluation of wire insulations at ambient temperature and 150 degrees celsius. Four samples of each specimen were tested using weights of one, two and three pounds at each temperature rating. Failure was determined by the abrading rod making electrical contact with the conductor. The number of cycles to failure was recorded.

The results of the abrasion resistance test at ambient temperature are shown in Figure 5 and the results at 150 degrees Celsius are shown in Figure 6. At ambient temperature, the MIL-W-81381 withstood approximately seven times more cycles than the PFPI samples. With a 2 pound weight, the differential was three times and at 3 pounds the difference less than two times. At elevated temperatures, the MII-W-81381 withstood eighteen times more cycles as did the PFPI construction. With both the 2 and 3 pound weights, the differential was two times. The imperfections in the PFPI insulation made this test very difficult to perform. The abrading tool would catch the edge of a hole or crack and rip the insulation as opposed to cutting through it. Individual data points indicate that the PFPI construction has the potential of performing as well as MIL-W-81381 in this test, providing the imperfections in the insulation are removed.

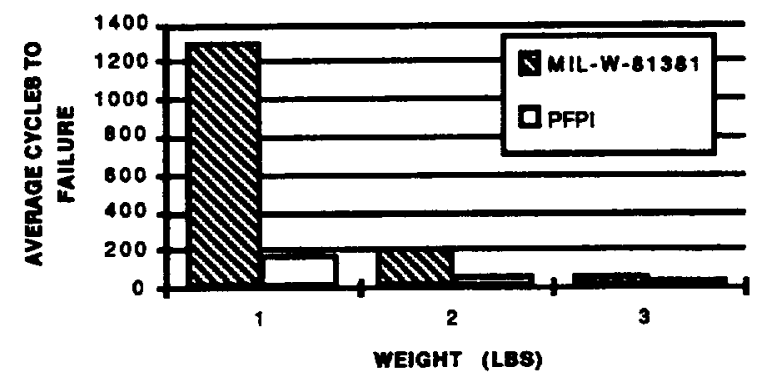

Figure 5. Abrasion Test Results at Ambient Temperature.

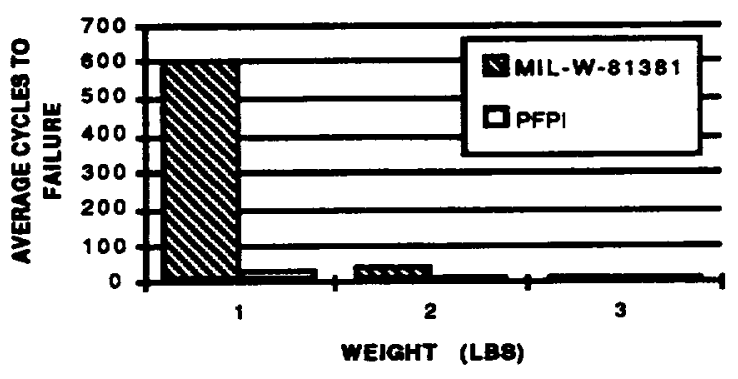

Figure 6. Abrasion Test Results at $150^{\circ} \mathrm{C}$.

\section{Dynamic Cut Through Test}

The dynamic cut through test was used to evaluate the resistance of the insulation system to penetration by a cutting surface at various temperatures. Eight samples of each wire type were tested for dynamic cut through at ambient, 70, 150 and 200 degrees Celsius. The cutting tool was a 20 mil tungsten carbide rod with a 4-6 mil finish. The test concluded when continuity was noted between the conductor and the cutting tool rod.

Results of the dynamic cut through test are shown in Figure 7. The MIL-W-81381 samples withstood approximately twenty percent greater force at each temperature tested. Part of the performance margin can be attributed to using stranded conductors in the MII-W81381 which permitted some compression of the wire and produced a greater surface area for the rod to penetrate.

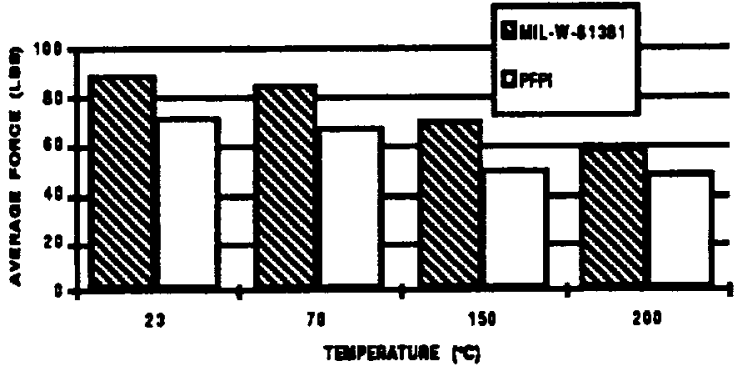

Figure 7. Dynamic Cut Through Test Results.

\section{Notch Propagation Test}

The notch propagation test was used to evaluate the ability of a wire insulation to withstand notching or nicking without propagating the damage down to the conductor. Six specimens of each sample were wrapped around a 0.25 inch mandrel at a rate of 30 revolutions per minute. One revolution was noted as the winding and unwinding of the wire in front of and then behind the notch. Any visual indication of bare conductor was 
considered a failure. After 100 cycles, the samples were submerged in a salt bath for one minute and subjected to a 2500 volt, $60 \mathrm{~Hz}$ voltage withstand test.

All six samples of the MII-W-81381 wire successfully passed the test. The bend radius of 0.25 inches was too severe for the PFPI construction. The insulation would crack and expose bare conductor as soon as the wire began wrapping around the mandrel. The insulation in the location of the notch remained in tact. Mandrel size was increased to determine the proper bend radius of the construction. With a 0.50 inch mandrel, the PFPI construction averaged 53.3 cycles before failure. Two samples broke in half and the voltage withstand test could not be performed, while the third sample passed. Cracking was noted away from the notch on all three samples. Wire breakage can be attributed to the flexing of the solid conductor and is not a characteristic of the insulation. With a 0.75 inch mandrel, the three samples successfully completed the 100 cycle requirement along with the voltage withstand test.

\section{Weight LosstOutgassing Test}

The weight loss under temperature and vacuum test was used to determine the weight loss of a preconditioned insulated wire when subjected to a vacuum at temperature. Six specimens of each sample were preconditioned at $95 \%$ relative humidity and 38 degrees Celsius for 72 hours. Another six specimens of each sample were preconditioned at less than 5\% relative humidity and 38 degrees Celsius for 72 hours. After preconditioning, the samples were weighed and placed in a temperature altitude chamber preconditioned to $\mathbf{2 0 0}$ degrees Celsius. The chamber was sealed and pressure was reduced to 33 Torr for 384 hours. At the completion of the 384 hours, the specimens were removed and weighed to acquire a weight loss (outgassing) value.

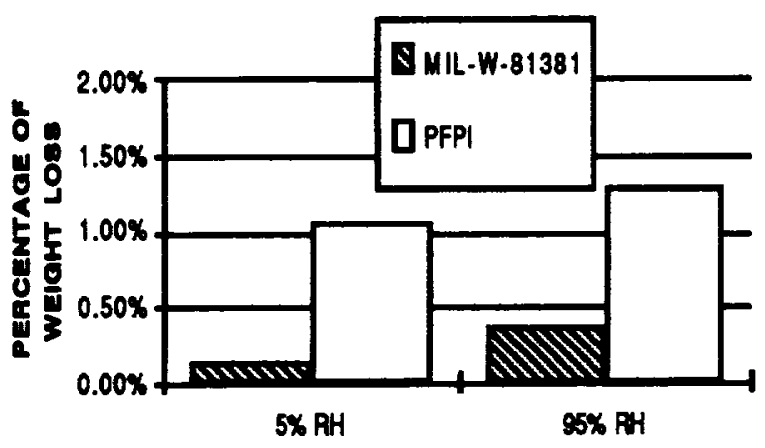

AELATIVE HUMOITY

Figure 8. Weight Loss/Outgassing Test Results.
The weight loss/outgassing results are shown in Figure 8. The PFPI insulated wire demonstrated a $0.9 \%$ greater weight loss than did the MIL-W-81381. Based on the percentage of weight gain/loss present in the PFPI material after preconditioning, moisture which was trapped in the imperfections of the insulation coating, was released during the test skewing the results.

\section{Conclusions}

The tests performed to date demonstrated the similar performance characteristics between MIL-W-81381 and PFPI insulations in the areas of AC corona inception and extinction and dynamic cut through. The MIL-W81381 construction outperformed the PFPI construction in the areas of wire fusing time, time/current to smoke, abrasion resistance, notch propagation and weight loss/outgassing. Based on the results of this evaluation, the present process of applying the PFPI coating to the conductor must be improved prior to conducting additional testing. The PFPI material demonstrated many outstanding qualities during this evaluation, but the presence of voids and imperfections in the PFPI coating negatively impacted its overall performance. Future evaluations of the PFPI material should focus on casting films which can be used in a multiple tape insulation construction.

\section{Acknowledgment}

The anthors would like to thank Dr. Daniel $R$. Mulville, Director of NASA HQ OS\&MA, Technical Standards Division for his continued support and technical guidance. The testing effort was performed by McDonnell Douglas Aerospace under contract NAS118580, Technology for Hyperspace Vehicles - Task \#12 Feasibility Study of New Wire Insulation Constructions for NASA Aerospace Wiring Applications. Work was also performed under contract NAS3-27186.

\section{References}

1. Soloman, R., Woodford, L., Domalewski, S., New Insulation Constructions for Aerospace Wiring Applications, McDonnell Douglas Aincraft Company, June 1991, WL-TR-91-4066.

2. Stavnes, M.W., Hammoud, A.N., NASA Requirements and Application Environments for Electrical Power Wiring, Sverdrup Technology, Inc., August, 1992, NASA CR-191064. 



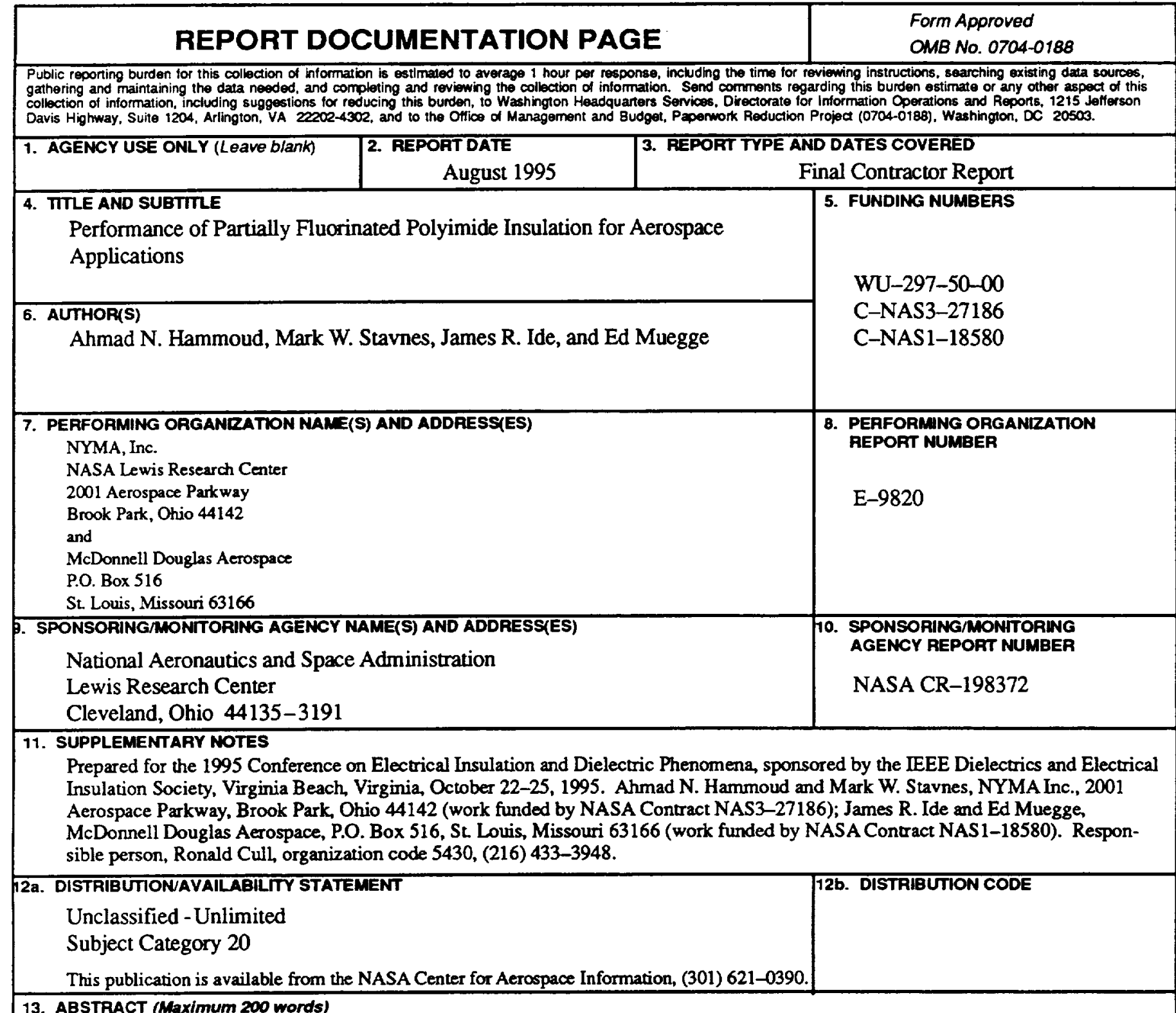

13. ABSTAACT (Maximum 200 words)

Polyimide has been used extensively as the primary wiring insulation in commercial planes, military aircraft and space vehicles due to its low weight, high service temperature and good dielectric strength. New failure modes, however, have been associated with the use of polyimide because of the susceptibility of the insulation to pyrolization and arc tracking. A new wiring construction utilizing partially fluorinated polyimide insulation has been tested and compared with the standard military polyimide wire. Electrical properties which were investigated include AC corona inception and extinction voltages (sea level and 60000 feet), time/current to smoke and wire fusing time. The two constructions were also characterized in terms of their mechanical properties including abrasion resistance, dynamic cut through and notch propagation. In this paper, these test efforts and the results obtained are presented and discussed.

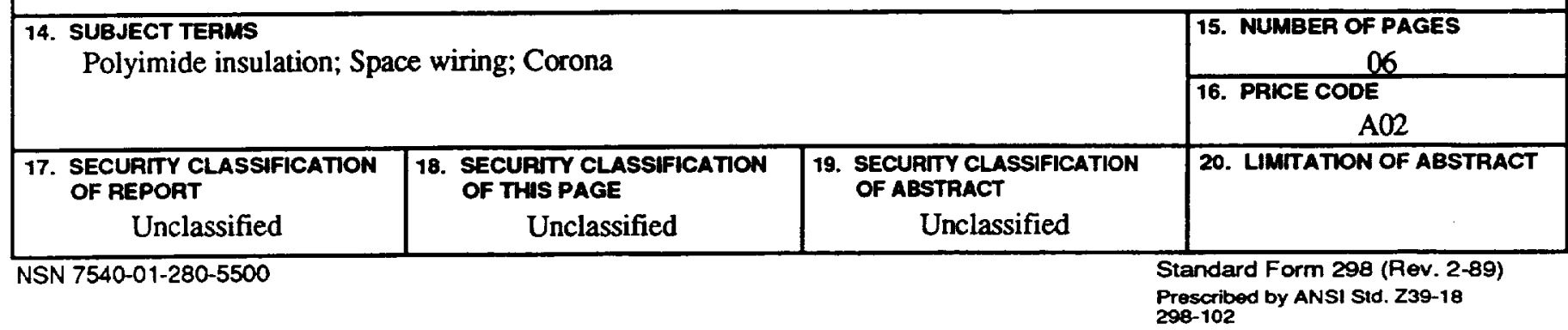

